\title{
An Efficient Optimization Technique for Node Clustering in VANETs Using Gray Wolf Optimization
}

\author{
Muhammad Fahad Khan ${ }^{1}$, Farhan Aadil ${ }^{1 *}$, Muazzam Maqsood ${ }^{1}$, Salabat Khan ${ }^{1}$, \\ and Bilal Haider Bukhari ${ }^{1}$ \\ ${ }^{1}$ Department of Computer Science, COMSATS Institute of Information Technology, \\ Attock, 43600 - Pakistan \\ E-mail: [m.fahad. farhan.aadil, muazzam.maqsood, salabat.khan, bukhari]@ciit-attock.edu.pk \\ *Correspondent Author: farhan.aadil@ciit-attock.edu.pk
}

Received February 12, 2018; revised March 30, 2018; accepted April 17, 2018;

published September 30, 2018

\begin{abstract}
Many methods have been developed for the vehicles to create clusters in vehicular ad hoc networks (VANETs). Usually, nodes are vehicles in the VANETs, and they are dynamic in nature. Clusters of vehicles are made for making the communication between the network nodes. Cluster Heads (CHs) are selected in each cluster for managing the whole cluster. This $\mathrm{CH}$ maintains the communication in the same cluster and with outside the other cluster. The lifetime of the cluster should be longer for increasing the performance of the network. Meanwhile, lesser the CH's in the network also lead to efficient communication in the VANETs. In this paper, a novel algorithm for clustering which is based on the social behavior of Gray Wolf Optimization (GWO) for VANET named as Intelligent Clustering using Gray Wolf Optimization (ICGWO) is proposed. This clustering based algorithm provides the optimized solution for smooth and robust communication in the VANETs. The key parameters of proposed algorithm are grid size, load balance factor (LBF), the speed of the nodes, directions and transmission range. The ICGWO is compared with the well-known meta-heuristics, Multi-Objective Particle Swarm Optimization (MOPSO) and Comprehensive Learning Particle Swarm Optimization (CLPSO) for clustering in VANETs. Experiments are performed by varying the key parameters of the ICGWO, for measuring the effectiveness of the proposed algorithm. These parameters include grid sizes, transmission ranges, and a number of nodes. The effectiveness of the proposed algorithm is evaluated in terms of optimization of number of cluster with respect to transmission range, grid size and number of nodes. ICGWO selects the $10 \%$ of the nodes as CHs where as CLPSO and MOPSO selects the $13 \%$ and $14 \%$ respectively.
\end{abstract}

Keywords: VANETs, LBF, Optimization, Intelligent Transportation Systems and clustering. 


\section{Introduction}

The collection of devices for the communication is called as the network. In networks, one of the mainstream lines is ad hoc network. However, ad hoc network focuses on two main directions known as mobile ad hoc network (MANETs) and VANETs. As proposed research has focused on VANETs so this paper will flow in the same direction. In VANETs the nodes are vehicles or automobiles on the roads. These vehicles combine to form a network. This network is created for the data sharing between the automobiles in the network [1]. There are huge number of applications of VANETs, for instance, entertainment, safety and emergency services and much more [2]. In VANETs, the communication is further divided into three categories. This division is based on the method of their working. These are Vehicle-to-Vehicle-based communication (V2V), Vehicle-to-Infrastructure (V2I) and last one is hybrid (V2V and V2I), which is the combination of first two categories.

In VANETs nodes can move here and there, so their movement is considered as random in motion. Due to which there is no fixed topology in VANETs. High mobility pattern leads to frequent changes in the topology. Consequently, this creates the problem of scalability in the VANETs, which is a vital issue in this domain. There are many proposed solutions while clustering is one of them. Clustering is the process of gathering the vehicles of the same vicinity. Clustering makes it easy to create the networks more optimized and scalable $[3,4]$. The clustering is considered as good enough for the resource utilization and LBF. Clustering isolates the whole network into small logical groups for increasing the life of the network. A mobility based clustering algorithm mobile ad hoc networking based clustering (MOBIC) is considered as the more steady in the domain of MANETs [5]. Clustering is the method of making logically groups of the network by some proper rule and regulation. There are different methods of clustering which is based on the variation in rules and regulations. On this variation the performance also varies from each other [6]. There is always a $\mathrm{CH}$ in each cluster, all other nodes in the cluster are called as cluster member or cluster nodes. CHs is responsible for the formation of the cluster, maintenance of network topology and distributing resources to all the nodes in the cluster. In a cluster, from all the members' one of the node is selected or nominated as $\mathrm{CH}$ for that specific cluster. There are many techniques for electing the $\mathrm{CHs}$. In some method every single node can be selected as $\mathrm{CH}$, However, in many other methods, CHs are taken by different properties of the nodes and their different parameters $[4,5,7]$.

Another issues also raise here is what will be the size of the cluster, which is totally dependent on the transmission range, due to which size of the clusters varies from each other $[4,8,9]$. Clustering is also considered as an NP-hard problem [10]. The meta-heuristics algorithms can be used to find the optimal solutions for different problems. In [11] research studies are tailored to explain the differences among various networks and their relative challenges. Cluster stability is a primary objective of the proposed algorithm to attain it. Consequently, stability plays a vital role in the communication between the upper and lower layers. This will increase the performance by using the clusters. Cluster stability can also be understood in various ways regarding parameters [6, 12];

i) Ratio of changes of CHs.

ii) The number of Cluster Nodes $(\mathrm{CN})$ changing the $\mathrm{CH}$.

First, is the rate of $\mathrm{CH}$ changing, while second shows the ratio of $\mathrm{CN}$ exchaning their $\mathrm{CH}$ with passage of time. 




Fig. 1. Clustering in VANETs [13]

\section{Clustering in VANET}

\subsection{Cluster Structure}

The structure of cluster is defined by the divison of nodes. The divison is based on the certain rule to create the virtual groups. These virtual groups of nodes contains the various status to show the significance of node in the cluster. These status can be as a gateway node of the cluster, managing node of the cluster and can be a simple member of the cluster. A managing node $(\mathrm{CH})$ is basically most important status in the cluster. These nodes are responsible for the management of resoucres in the cluster. Resources of clusters can be used for the inter and intra-cluster communication. However gateway nodes deals with the inter-cluster transmission of data.

\subsection{Clustering in VANETs}

Since decades VANETs is considered as the exploroational topic in the research. Consequently, clustering in VANETs is also a emerging filed in the networks domain. The reason of clustering in VANETs contains; togather the nodes in one vicinity to stabilize the dynamic nodes, optimizing the resources utilization for the routing, rapaid convergence with reducing the overhead, optimizing the power consumption, Scalibility of network is also increased by cluserting by reduing the overhead. Minimun the amount of overhead for the $\mathrm{CH}$ to manage, the network will be more scalable and stable to manage. The stability of network also enforce for the efficient transmission of data in VANETs. It also leads the network, to enforce for reducing the fluctuation rate of network topology. Moreover, it also helps to manage the larger network easily. 
The range of clusters can be varied based on the transmission range of the nodes. The Fig. 1. represents the different clusters having different ranges and their $\mathrm{CHs}$, cluster members and cluster gateways.

Research has shown that routing on the top of clustering architectures is more scalable and stable as compared to flat routing [14-17].

\subsection{Aggregate Local Connectivity}

In this method the clustering is applied on each node by calculating the number of neighbors. This is computed by aggregating the total number of ping/beacon messages received by a node with a specific time interval. There is also a threshold value $(\lambda)$ which is started from null value and considered as the current minimum value. By using number of iterations the cluster matrix is created. Also it is focused so that only one node is selected in not more than one cluster. $\mathrm{CH}$ is also selected from the cluster matrix by conisdering the factor of connectivity of nodes which is called as Aggregated Degree Connectivity.

Aggregated Degree Connectivity (ADC) is termed as $\mu$, which shows the value of a node association with the neighbors. Larger the value of connectivity of a node with the neighbors, greater will be the chances of a node to be selected as $\mathrm{CH}$. The selection process of a $\mathrm{CH}$ is also dependent on speed, direction of nodes, vicinity of nodes and transmission range along with the ADC. This factor helps the ICGWO to select the most appropriate nodes as the cluster member and $\mathrm{CH}$, which increases the network lifetime.

\subsection{Benefits of Clustering in VANETs}

There are several advantages of clustering in VANETs, some of them are as follows;

Clusteirng provides the better usage of resources and control the topology effectively. The same frequency is used by the clusters in case of non-overlapping.

$\mathrm{CHs}$ are responsible to manage the bandwidth allocation, especially when collision of transmission occurred.The path for the transmission of inter-cluster communication is establised with the help of CHs of different clusters. Clustering helps the mobile nodes to look as a stable nodes, due to which resources are also properly managed in the network.

In case of mobility the corresponding node only exchange the information with the $\mathrm{CH}$ of adjacent cluster $[15,17,18]$. Fig. 1. shows the cluster of vehicles communicating with each other. The red circled area depict the range of $\mathrm{CH}$.

To the best of mine knowledge, the proposed scheme is a novel method that uses proposed methodology for very first time in the domain of VANET environment for the clustering. Moreover, weightage is assigned to each objective as per the user requirements. Furthermore, each step of the proposed work is statistically modeled for a detailed explanation. At the end, comparison of new technique is held with other popular methods.

\section{Literature Review}

The major purpose of VANET deployment is enabling vehicular communication for special purposes such as reporting traffic conditions, driver's and passenger's conditions, sending emergency and collision warnings, monitoring roads surfaces and weather conditions, data sharing, and other safety-related purposes, just to mention a few [19]. VANET is the principal framework for intelligent transportation systems (ITS). ITS is proposed with the purpose of 
designing vehicle operations, assisting drivers to obtain needed information for safety and entertainment purposes, traffic management, and providing convenience for passengers. ITS is expected to grow as its ultimate goal is the realization of a safe and accident-free driving environment. Automatic toll collection and driving assistance systems may be cited as examples. ITS applications generally require numerous messages being transferred via multiple hops between vehicles to travel from source to destination.

Gerla and Tsai [7], proposed the clustering algorithm which is based on the highest connectivity. In this method, the degree of the node is calculated, and the node with the maximum degree is selected as the $\mathrm{CH}$. Genetic algorithm based clustering algorithm is proposed by S.K. Das et al. [8]. Chatterjee et al. [20] proposed the framework weighted clustering algorithm (WCA) where weights are assigned to the required objectives. Weights are assigned by calculating the different parameters. Shahzad et al. [21] proposed the framework for the clustering in MANETs known as CLPSO. This algorithm efficiently minimizes the number of required cluster for the communication. This communication is for inter and intra-cluster transmission of data. The key parameters used in the CLPSO are battery power, transmission range, ideal degree and node mobility. There is another method in which clustering problem in VANETs is solved by using the meta-heuristics algorithms.

Laing et. al. [22] proposed the techniques for the device to device (D2D) communication in the environment of ultra-dense networks. Proposed framework gave the robust and flexible results in the dynamic wireless networks for the scale video coding with fountain coding. Afterwards, proactive and active based method is introduced for the content up to date. Meanwhile Laing et. al. [23] also proposed the another method for the D2D communication in smart cities. In this method the investigation was carried to show the relation between the between coding, storage and transmission.

Han et. al. [24] proposed the technique to minimize the cost by considering the parameter of average delay time. The Lynapunov optimization algorithm is used to develop an optimal solution for large data. Afterwards the Han Hu et. al. [25] used the same methods and environment to satisfy the Quality of Service requirements. The proposed algorithm gave the better results as compare to traditional methods.

Farhan et al. [26] proposed the Ant Colony optimization (ACO) based clustering algorithm for the VANETs called as CACONET. This method gave the optimal number of cluster for the efficient communication in the VANETs. Another technique is also known as Ant Colony based Intelligent Clustering in vehicular ad hoc networks (ACONET) proposed by Khan at el. [27] which is also based on the social behavior of ants. In this work, the entire nature of ants is implemented in the proposed frame for solving the mentioned problem.

Baker at el. [28] also gave the solution for finding the suitable cluster. Each node has unique ID, and the node had the lowest ID in the cluster will be selected as the CH. Hamid at el. [11] proposed the algorithm, MOPSO in which multiple results are extracted at the output for the one problem. The best one according to the problem can be used. Multi-Objective Problems evolutionary algorithm is best enough for finding the multiple solutions. This algorithm is developed to obtain more than one solutions for a single problem [29]. Other evolutionary algorithms are implemented for optimized clustering [8, 11, 21, 30], so this encouraged us to employee GWO based algorithm named ICGWO. In 1995 James Kennedy and Eberhard proposed the algorithm Particle Swarm Optimization (PSO) [31]. In this method, individuals 
in the flock find their personal best and global best. By finding the values, the whole flock converges toward the global best value for finding the target. GWO is also using for the solving of different problems, experienced Gray Wolf Optimizer [32] is used with the reinforcement learning in neural network method to enhance the performance.

The main idea behind MOBIC is to compare nodes with their neighbors based on their mobility metrics and to add them to appropriate clusters. A node with lowest relative mobility compared with its neighbors is selected as $\mathrm{CH}$. A CH with high relative mobility compared to its neighbors results in poor cluster stability. The mobility metric proposed in MOBIC does not require location information about nodes. Relative mobility is calculated based on received signal strength of two consecutive messages from the same neighbor node. MOBIC is a weight based and one-hop clustering protocol. The clustering scheme used for MOBIC is similar to lowest ID algorithm [7]. A notable property of MOBIC includes the merging process of two clusters. When two CHs meet, the merging time is postponed for CCI time interval. The CCI or cluster contention interval is introduced as a waiting time for cluster merging process. After this waiting time if two CHs are still in each other's range, their clusters are supposed to merge and the one with lowest ID takes over the $\mathrm{CH}$ responsibility. The evaluation results represent a better performance of MOBIC in terms of $\mathrm{CH}$ changes because of using relative mobility instead of node ID.

\section{Proposed Technique}

\subsection{Gray Wolf Optimizer}

Gray wolf (Canis lupus) belongs to Canidae family. The specification of wolves is sharp teeth, bushy tail. The group of gray wolves is 5-12. They are habitats of mountains, forests, etc. These are considered at the top of the food chain and known as the apex predator. The social behavior of GWO is extracted which is entirely based on the few steps/phases searching, encircling and hunting. In nature, the GWO relies on the four positions called as alpha $(\alpha)$, Beta $(\beta), \operatorname{delta}(\delta)$ and Omega $(\omega)$. As shown in Fig. 2. given below.,

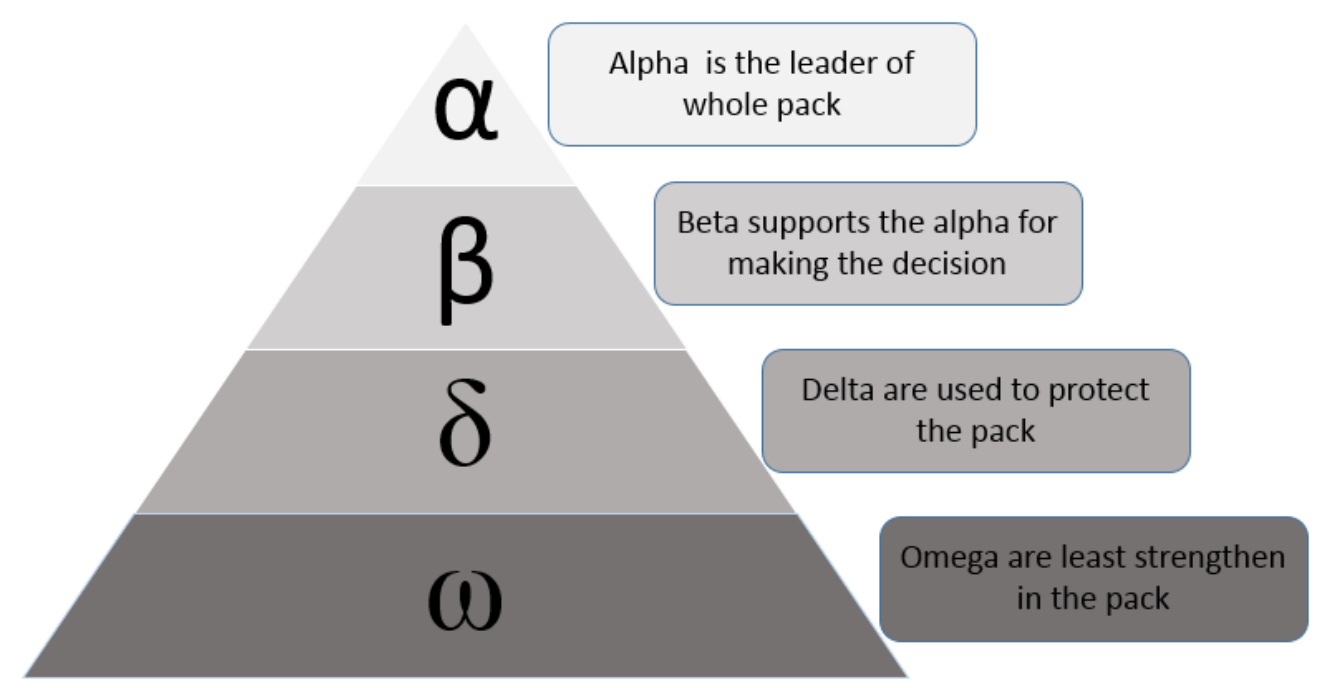

Fig. 2. Complete Hierarchy of Gray Wolves 
In the given pyramid the alpha is considered as the strongest participant in the pack. Alpha $(\alpha)$ is at the top of the hierarchy, and considered as the strongest candidate in the pack. Alpha is normally male wolf but can be female as well. Alpha wolves gave the order, which is followed by all the other wolfs in the pack. Beta wolves are usually responsible to implement the orders of alpha. Alpha wolves also search for the sleeping place for the pack [33].

Afterward, beta gray wolves play an imortant role in the hirarchy. These are the second most important wolves in the pack. Alpha wolves take the decisions with the help of beta wolves. The beta wolves also coordinate in the feedback pupose. Subsequently, delta wolves comes and categarize as gurads, predators, caretaker and spies. Next is the position of omega wolves. These wolves are considered as babysitters and are allowed to eat in the last.

Third order of gray wolves is delta. These wolves also have categories spies, guards, predators, and caretakers belong to Deltas. These golf help to protect the complete pack, also they keep eyes on the boundaries so that in the case of danger some measures can be taken for the pack. Hunters provide the food for the others, and caretakers look after the aged, weak and sick wolves in the pack.Omega exists in the last position of gray wolves. Due to the last in the position of wolves, they always have to pay a lot in return for the very small reward. Omega wolves also seem as babysitters, with no importance individually in the pack but the problem occurs after in case of losing these wolves. They are allowed to eat lastly after hunting.

In proposed methodology, Alpha $(\alpha)$ are considered as the best solution for the problem. If any solution which belongs to alpha, is not considerable due to the randomness of problem then the next most appropriate solution from the level beta, will be considered as the fittest solution.

Table 1. Actions of GWO.

\begin{tabular}{|c|c|c|}
\hline Step 1 & $\begin{array}{l}\text { 1. Tracking } \\
\text { 2. Chasing } \\
\text { 3. Approaching the prey }\end{array}$ & $\begin{aligned} \vec{X}(t+1) & =\overrightarrow{X_{P}}(t)-\vec{A} \cdot \vec{D} \rightarrow(1) \\
\vec{X}(t+1) & =\frac{\left.\overrightarrow{\left(X_{1}\right.}+\overrightarrow{X_{2}}+\overrightarrow{X_{3}}\right)}{3} \rightarrow(2)\end{aligned}$ \\
\hline Step 2 & $\begin{array}{l}\text { 1. Pursuing } \\
\text { 2. Encircling } \\
\text { 3. Harassing the prey }\end{array}$ & $\vec{D}=\left|\vec{C} \cdot \overrightarrow{X_{P}}(t)-\vec{X}(t)\right| \rightarrow(3)$ \\
\hline Step 3 & 1. Attack the prey. & $a=2-1 *\left[\frac{2}{\text { Max }_{\text {iter }}}\right] \rightarrow$ (4) \\
\hline
\end{tabular}

Where;

$\mathrm{A}$ and $\mathrm{C}$ are co-efficient vectors, $\mathrm{Xp}$;Position vector of prey, 
$\mathrm{X}$;Position vector of Gray Wolves.

The vector $\vec{A}$ and $\vec{C}$ is;

$$
\begin{gathered}
\vec{A}=2 \vec{a} \cdot \overrightarrow{r_{1}}-\vec{a} \rightarrow(5) \\
\vec{C}=2 \cdot \overrightarrow{r_{2}} \rightarrow(6)
\end{gathered}
$$

$\overrightarrow{r 1}$ and $\overrightarrow{r 2}$; random vector [0 to 1$]$.

Whereas;

$\overrightarrow{\boldsymbol{a}}$;linearly decreasing factors [2 to 0]. Fig. 3(a,b). is used to show the 2 and 3-dimensional view of the wolfs and prey. It shows all the expected positions of the gray wolf with respect to the movement of prey. Fig. 4. is also used to show the position updation of wolfs with respect to the target.

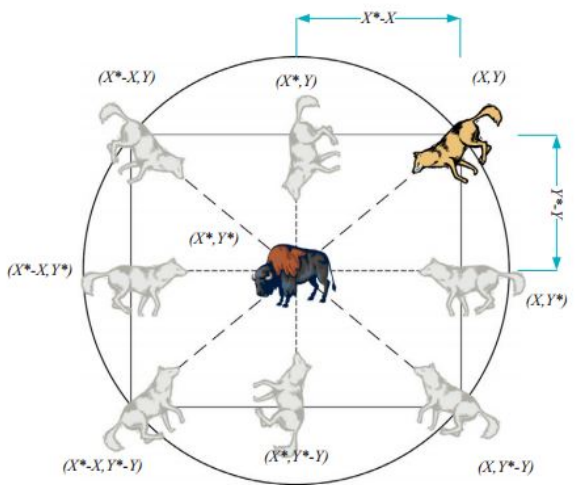

(a)

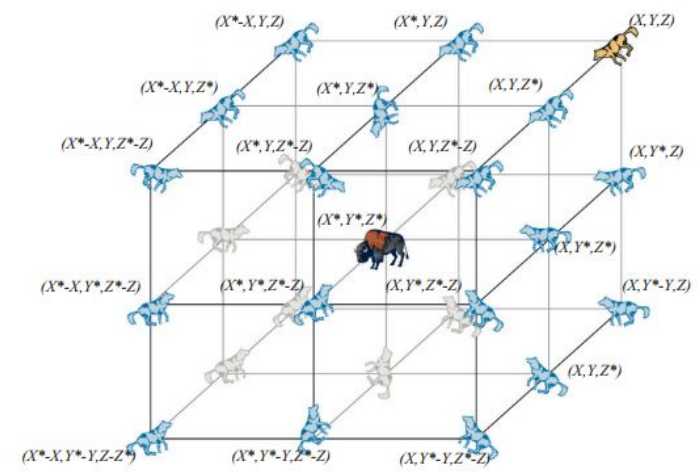

(b)

Fig. 3. Possible Position of wolf w.r.t Prey
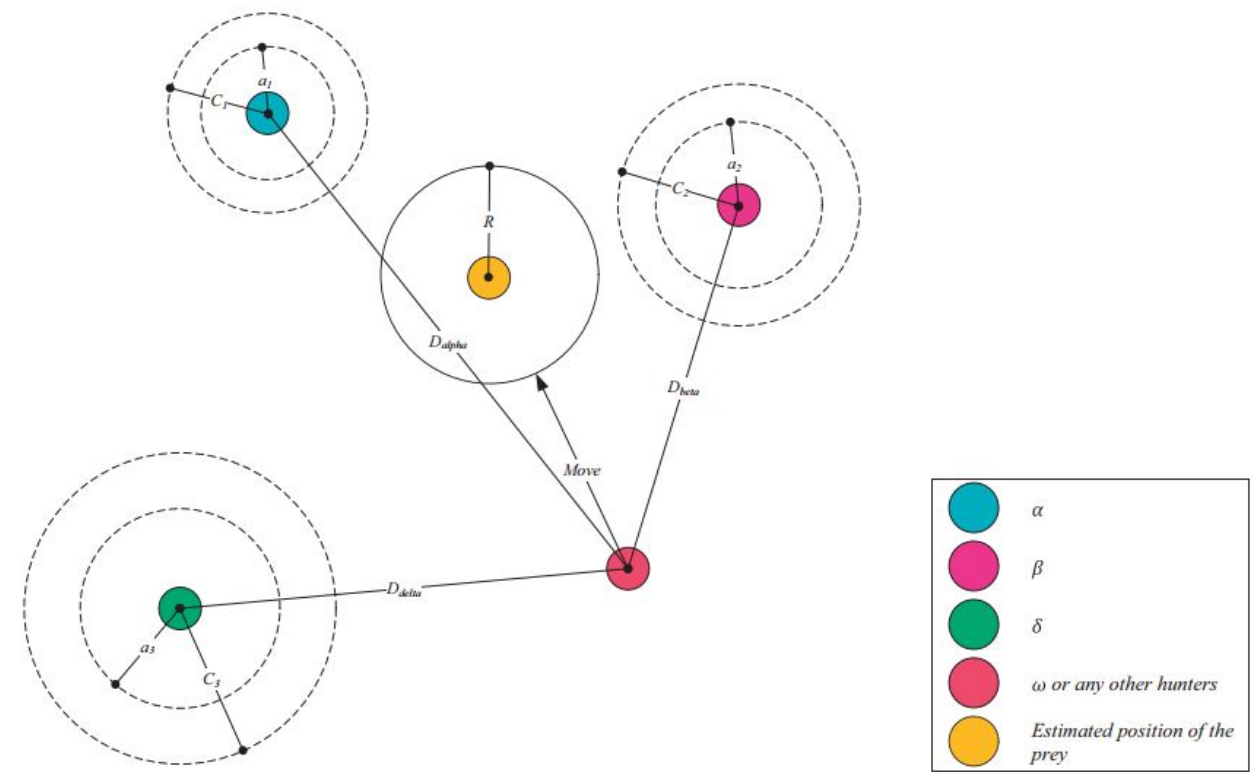

Fig. 4. Position updating in ICGWO 


\subsection{ICGWO Pseudo Code}

In the given solution the complete hierarchy of ICGWO is implemented for solving the discussed problem. The search agents in the three dimesion are deployed randomnly with in the required grid size. The Eucliadian distance between each search agents is also measured and called as neighbor distance. By using this distance the clustering technique known as Aggregate Local Connectivity is used to find the number of solutions. After getting the number of solutions, fitness function is used with the help of variant of GWO called as ICGWO. By using ICGWO the three main fitness values and positions are obtained. As, alpha is considered the fitter value in GWO so the number of required solution given by the alpha is taken as the optimized value of clusters for the specific suitaion, for which experiments have performed. The beta is considered the second most suitable value and respectively delta. As, omega value is not calculated because these delta wolf does not contribute in hunting. Therefore, these are not considered as the better solution.

\section{1: Begin}

2: Randomly deploy the vehicles on the highway.

3: Assign the velocity to all vehicles.

4: Randomly set vehicle's direction.

5: Assign the Vehicle-ID by creating the mesh topology.

6: Compute the distance between vehicles, and create the distance matrix.

7: Initialize a, A and C by using the Equation (5) \& (6)

9: WHILE (Num_Iteration == Total_Iterations)

10: Calculate the fitness of each search agent

i. $\quad \mathrm{X} \alpha=$ the best search agent

ii. $\quad X \beta=$ the second best search agent

iii. $\quad \mathrm{X} \delta=$ the third best search agent

11. WHILE ( $\mathrm{t}<$ Max number of iterations)

12. FOR each search agent

i. Update the position of the current search agent by using the Equation (1) \& (2)

\section{End FOR}

14. Update a, A and C by using the Equation (5) \& (6)

15. Calculate the fitness of all search agents

16. Update $\mathrm{X}_{\alpha}, \mathrm{X}_{\beta}$ and $\mathrm{X}_{\delta}$ i. $\mathrm{t}=\mathrm{t}+1$

a. IF (Best-Wolfs-cost $==$ Last iteration Best-Wolfs-cost)

i. Stall-Iteration ++;

\section{b. ELSE}

ii. Stall-Iteration $=0$;

c. END IF

d. Iteration++;

17: END WHILE

18: CHs $=$ Best-Wolfs- $\mathrm{X} \alpha$;

19: End 


\subsection{Block Diagram}

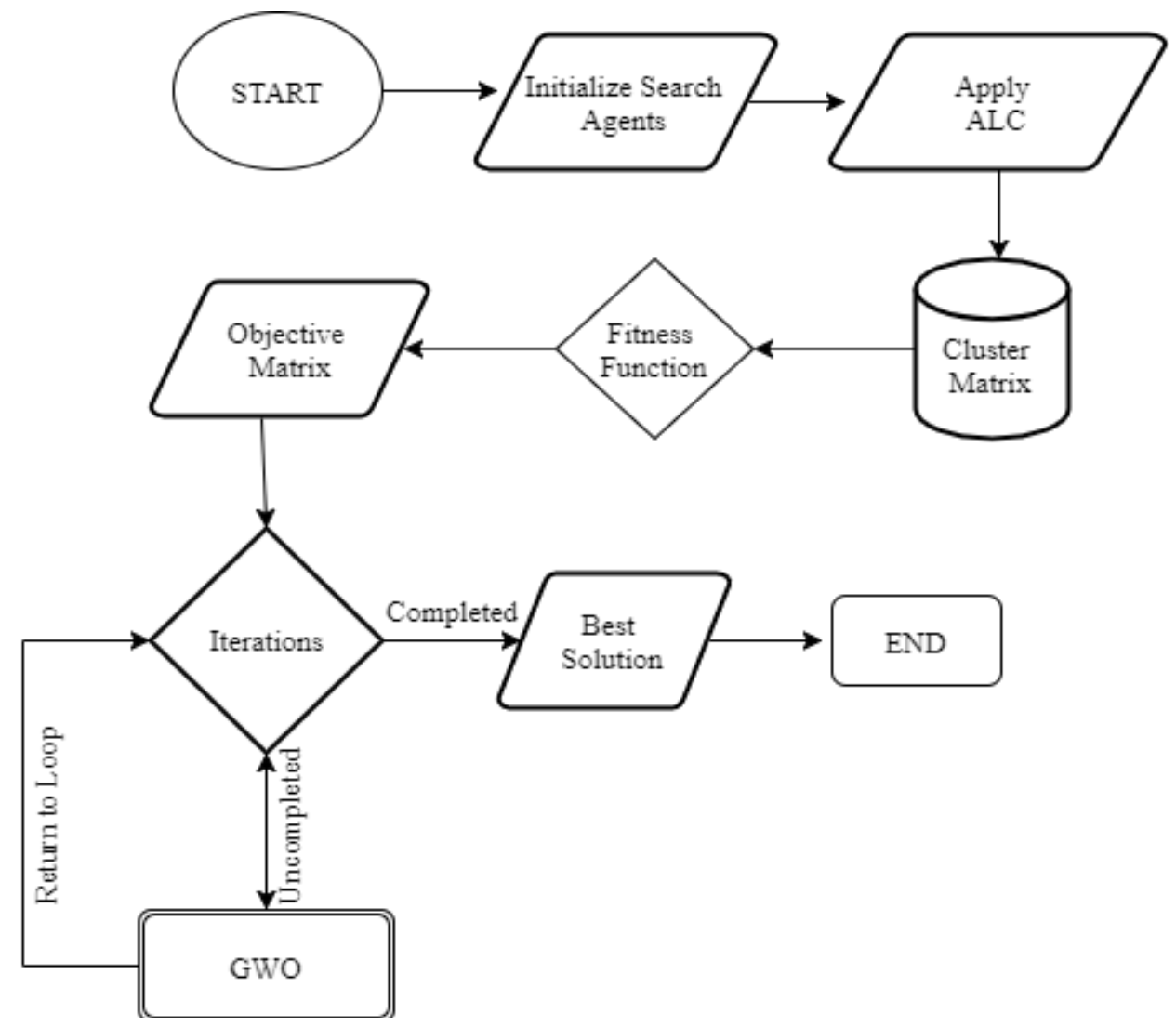

Fig. 5. Block Diagram of ICGWO

\section{Experimentations, Results and Discussions}

The experiments are described in this section. The proposed framework is implemented in the matrix laboratory R-2015a. After implementation of the novel technique, comparative analysis of ICGWO is held with the well-known meta-heuristics algorithms i.e. CLPSO and MOPSO. The results are professionally shown in three-dimensional (3-D) for the better understanding of the outcomes. The complete working and flow of proposed method is shown in Fig. 5. The given results show that the proposed method is showing the minimum number of required clusters as compare to others. This reduction in the required number of clusters will lead us to reduce the required resources for managing the network. This will reduce the routing cost, the number of hop of the network. Due to less number of clusters, the packet delays will be minimized as well.

The parameters used in the simulation are mentioned in Table 2. 
Table 2. Simulation Parameters for ICGWO,CLPSO and MOPSO

\begin{tabular}{|c|c|c|c|c|}
\hline $\begin{array}{l}\text { Sr. } \\
\text { No. }\end{array}$ & Parameters & ICGWO & CLPSO & MOPSO \\
\hline 1 & Population-Size/Particles & 100 & 100 & 100 \\
\hline 2 & Maximum-Iterations & 150 & 150 & 150 \\
\hline 3 & Inertia-Weight (W) & 0.649 & 0.649 & 0.649 \\
\hline 4 & $\mathrm{C}_{1}^{1}$ & 2 & 2 & 2 \\
\hline 5 & $\mathrm{C}_{2}{ }^{1}$ & 2 & 2 & 2 \\
\hline 6 & Simulation area & $\begin{array}{l}100 \times 100 \mathrm{~m}^{2}, \\
200 \times 200 \mathrm{~m}^{2}, \\
300 \times 300 \mathrm{~m}^{2}, \\
400 \times 400 \mathrm{~m}^{2}\end{array}$ & $\begin{array}{l}100 \times 100 \mathrm{~m}^{2}, \\
200 \times 200 \mathrm{~m}^{2}, \\
300 \times 300 \mathrm{~m}^{2}, \\
400 \times 400 \mathrm{~m}^{2}\end{array}$ & $\begin{array}{l}100 \times 100 \mathrm{~m}^{2}, \\
200 \times 200 \mathrm{~m}^{2}, \\
300 \times 300 \mathrm{~m}^{2}, \\
400 \times 400 \mathrm{~m}^{2}\end{array}$ \\
\hline 7 & Lower-Bound (lb) & 0 & - & - \\
\hline 8 & Upper-Bound (ub) & 100 & - & - \\
\hline 9 & Dimensions (Dim) & 3 & - & - \\
\hline 10 & Transmission range & 10 to $60 \mathrm{~m}$ & 10 to $60 \mathrm{~m}$ & 10 to $60 \mathrm{~m}$ \\
\hline 11 & Mobility Models & $\begin{array}{c}\text { Freeway Mobility } \\
\text { Model }\end{array}$ & $\begin{array}{l}\text { Freeway mobility } \\
\text { model }\end{array}$ & $\begin{array}{c}\text { Freeway mobility } \\
\text { model }\end{array}$ \\
\hline 12 & Simulation runs & 10 & 10 & 10 \\
\hline 13 & $\mathbf{W}_{1}$ & 0.5 & 0.5 & 0.5 \\
\hline 14 & $\mathbf{W}_{2}$ & 0.5 & 0.5 & 0.5 \\
\hline 15 & Nodes & $30,40,50,60$ & $30,40,50,60$ & $30,40,50,60$ \\
\hline 16 & Vehicle's velocity range & $22 \mathrm{~m} / \mathrm{s}-30 \mathrm{~m} / \mathrm{s}$ & $22 \mathrm{~m} / \mathrm{s}-30 \mathrm{~m} / \mathrm{s}$ & $22 \mathrm{~m} / \mathrm{s}-30 \mathrm{~m} / \mathrm{s}$ \\
\hline 17 & Maximum acceleration & $1.5 \mathrm{~m} / \mathrm{s}^{2}$ & $1.5 \mathrm{~m} / \mathrm{s}^{2}$ & $1.5 \mathrm{~m} / \mathrm{s}^{2}$ \\
\hline 18 & $\begin{array}{l}\text { Minimum Distance in } \\
\text { Vehicles }\end{array}$ & - & $2 \mathrm{~m}$ & $2 \mathrm{~m}$ \\
\hline 19 & $\begin{array}{l}\text { Maximum Distance in } \\
\text { Vehicles }\end{array}$ & - & $5 \mathrm{~m}$ & $5 \mathrm{~m}$ \\
\hline 20 & Width of Lane & - & $50 \mathrm{~m}$ & $50 \mathrm{~m}$ \\
\hline 21 & Num of Lanes & - & 8 & 8 \\
\hline
\end{tabular}

Learning Factor ${ }^{1}$ 




Fig. 6. Number of clusters vs Number of nodes vs Transmission range in ICGWO, MOPSO and CLPSO by fixing nodes from 30 to 60 , for grid size $=100 \mathrm{~m}$

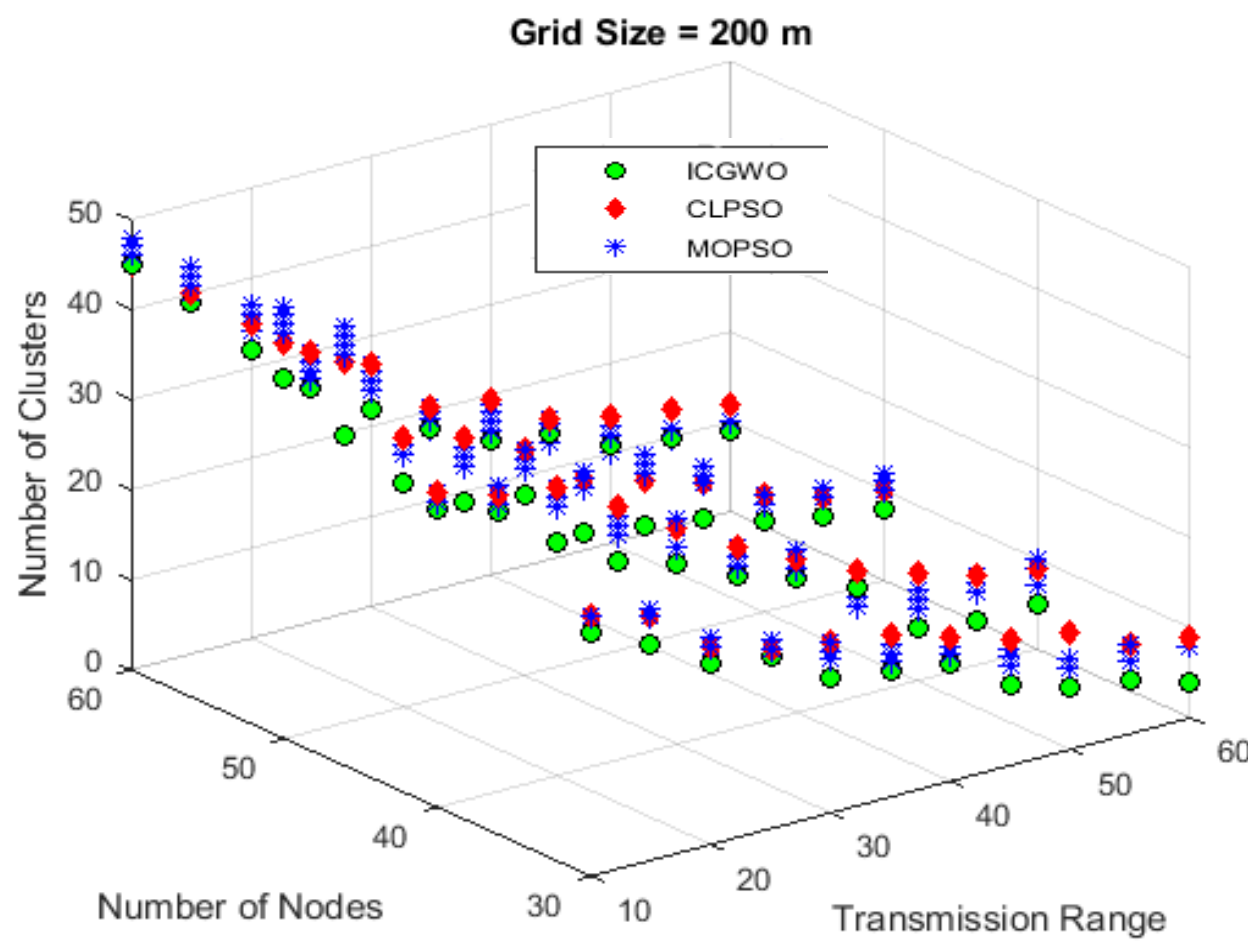

Fig. 7. Number of clusters vs Number of nodes vs Transmission range in ICGWO, MOPSO and CLPSO by fixing nodes from 30 to 60 , for grid size $=200 \mathrm{~m}$ 


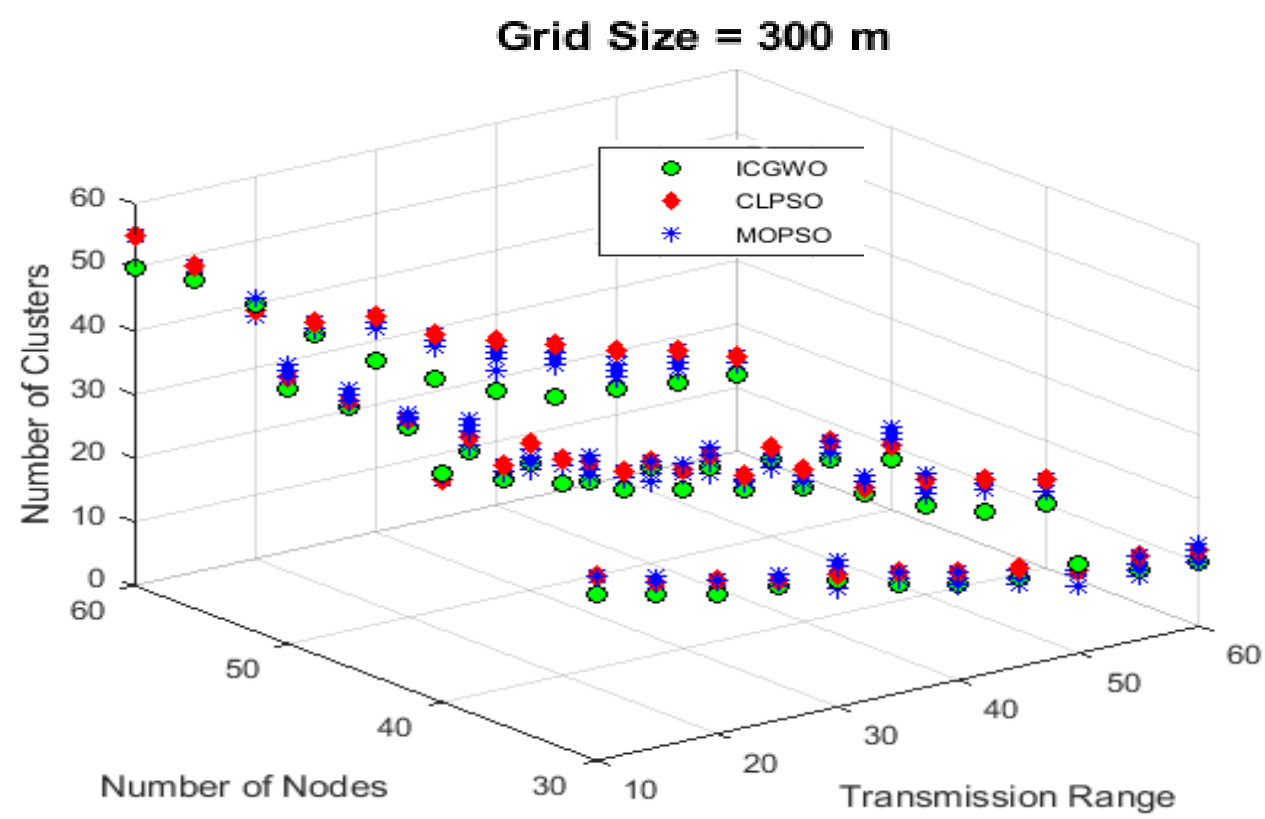

Fig. 8. Number of clusters vs Number of nodes vs Transmission range in ICGWO, MOPSO and CLPSO by fixing nodes from 30 to 60 , for grid size $=300 \mathrm{~m}$

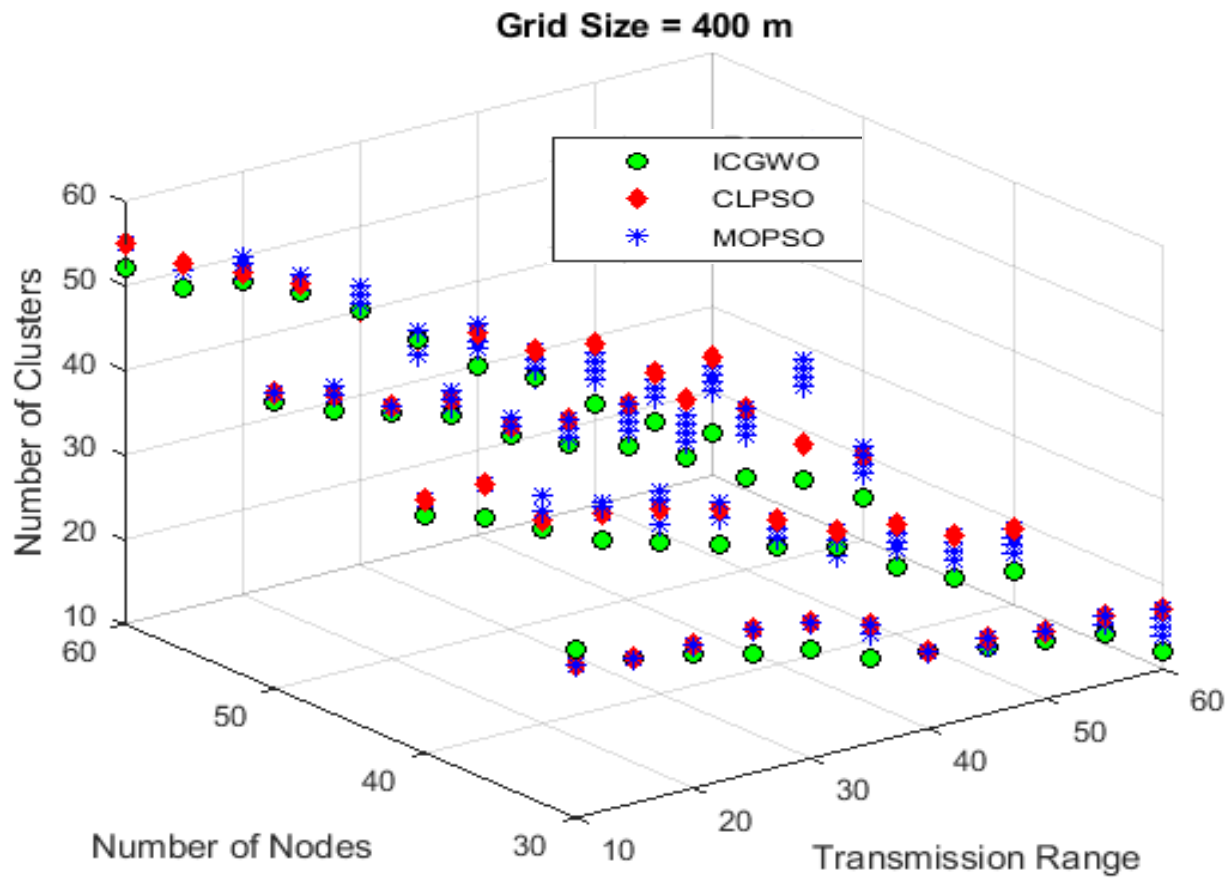

Fig. 9. Number of clusters vs Number of nodes vs Transmission range in ICGWO, MOPSO and CLPSO by fixing nodes from 30 to 60 , for grid size $=400 \mathrm{~m}$ 
The results are shown in the Fig. 6,7,8 and 9. Transmission Range in the $x$-axis, Number of nodes in $\mathrm{y}$-axis and number of clusters in the z-axis. The transmission range is from $10 \mathrm{~m}$ to 60 $\mathrm{m}$, the number of nodes are 30-60 while different grid size from $100 \mathrm{~m}$ to $400 \mathrm{~m}$ is used to show the required number of cluster accordingly. The ICGWO shows the optimized number of clusters is as shown in the figures represented with green filled circle. The number of required clusters are inversely proportional to the transmission range. When the value of transmission range is increased, the required number of clusters will be decreased. We can see that ICGWO is showing the optimized results as compare to CLPSO and MOPSO in all the given scenarios. The size of the grid is also changed to make the results more strong and perfect. Graphs illustrate the results favorable to the ICGWO. Also, the number of nodes/vehicles are changed so that the accuracy of the proposed method can be measured. At some point in the network, MOPSO overlaps with the proposed method. But this is due to the randomness nature of the algorithm. The results are taken after the ten iterations for each scenario and then the average value is taken to plot the results. Even though MOPSO provides the multiple solutions for the problem but still ICGWO is providing the optimized results for the given situation.

\section{Computational Complexity}

Following symbols are used in calculations:

$\mathrm{z}=$ number of gray wolves

$r=$ total number of iterations executed

$\mathrm{n}=$ total number of vehicles/nodes

$\mathrm{k}=$ Average number of CHs in a solution constructed by ant.

The complexity of ICGWO is calculated in small steps and then merge together to show the overall complexity.

\subsection{Solution construction by a single wolf}

$\mathrm{O}$ (n) time is required to add the $\mathrm{CH}$ in the solutions, for the ICGWO. Probability calculations is exceuted for the exploration and exploitation. The calculation is performed ' $k$ ' times to make the decision. Therefore, $\mathrm{O}(\mathrm{n})$ is required to construct the solution.

\subsection{Solution Quality / Fitness}

As per the discussion, the ' $\mathrm{i}$ ' number of clusters head for a solution consume $\mathrm{O}$ (i.n) time for the fitness value.

\subsection{Searching, Encircling and Attacking}

ICGWO takes $\mathrm{O}$ (i) time to explore the search space for finding the best solution between the ' $\mathrm{i}$ ' clusters heads associated to the result. It revenues $\mathrm{O}(\mathrm{n})$ time to fitter solution out of the alpha, beta, delta and omega or on unused CHs. Since $\mathrm{k}<=\mathrm{n}$ with trend to less, this adds-up to $\mathrm{O}$ (n) for ICGWO. ICGWO entails $\mathrm{O}\left(\mathrm{n}^{2}\right)$ jobs to the optimized number of clusters for the scenarios.

\subsection{Complexity of while loop (i.e. batch of gray wolves)}

ICGWO consumes O (i.n) + O (i.n) + O (n) for a wolf which falls to: O (i.n) and for ' $\mathrm{j}$ ' wolves, it converts $\mathrm{O}$ (j. (i.n)) 


\subsection{For ' $x$ ' rules creations in WHILE loop}

So the overall complexity of ICGWO is $\mathrm{O}\left(\mathrm{x} .(\mathrm{j} .(\mathrm{i} . \mathrm{n}))+\left(\mathrm{n}^{2}\right)\right)$, where $\mathrm{n}^{2}$ denotes exploration and exploitation operation.

\section{Load Balance Factor}

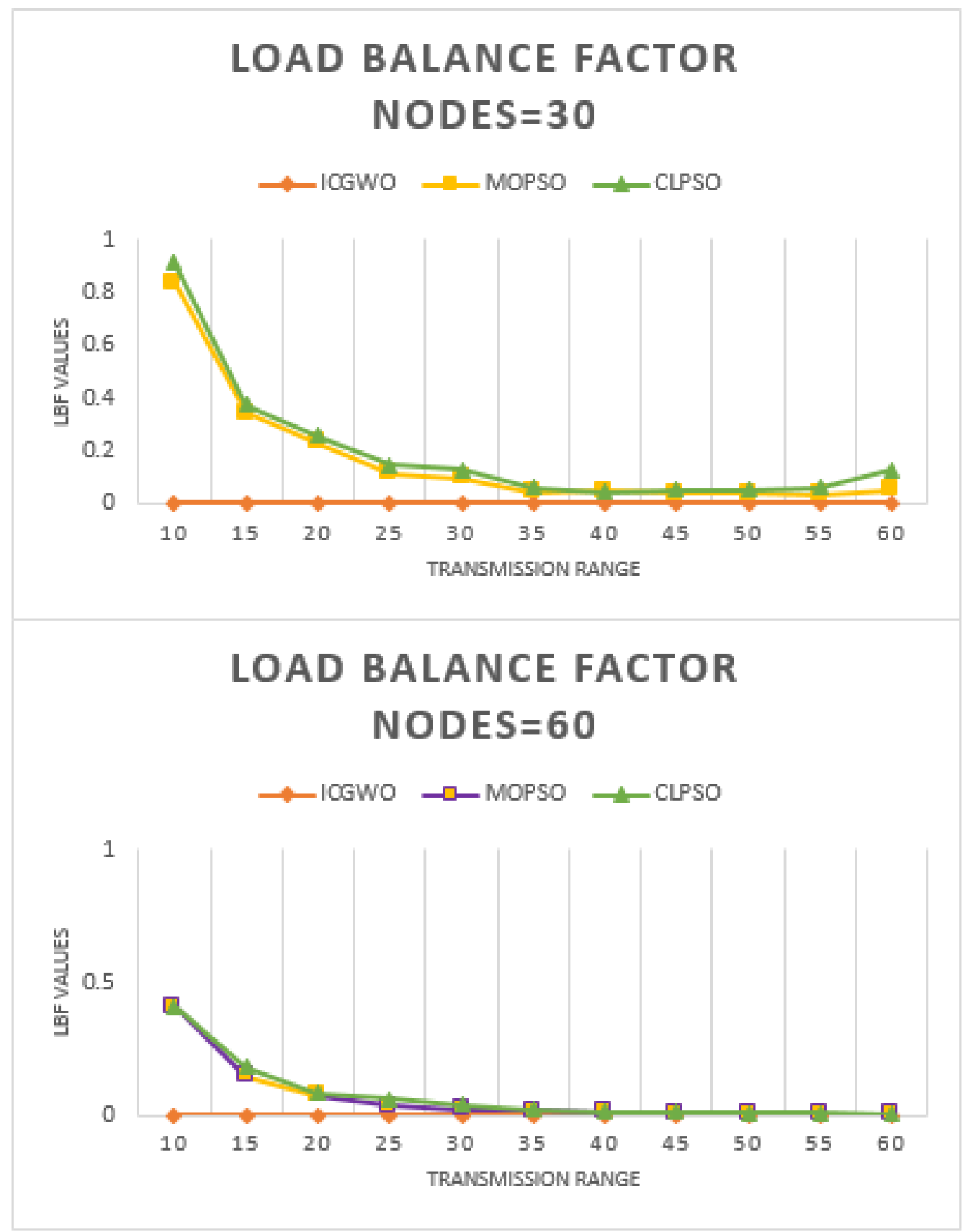

Fig. 10. LBF Vs Transmission Range for 100m X 100m 




Fig. 11. LBF Vs Transmission Range for 200m X 200m

LBF is used to evaluate the load on each CH. It is very difficult for each cluster to allocate the equal number of CNs. LBF is used for the balanced allocation of load in the cluster. The primary cause is due to the rapid variation of neighbors from the CHs. The cardinality of the cluster size represents the load of a CH. In [11], the LBF is defined as, 


$$
\text { Load Balance Factor }=1 /\left(n_{c} \times \sum_{i}\left(x_{i}-\mu\right)^{2}\right) \rightarrow(7)
$$

$\mathbf{n}_{\mathbf{c}}$ : Number of CHs.

$\mathbf{x}_{\mathbf{i}}$ : Load of cluster size.

i: $1,2,3 \ldots . . . n$.

$\boldsymbol{\mu}$ : Total Number of Nodes in the Network.

The Fig. 10. and 11. shows the LBF of mentioned algorithms, graphs shows that ICGWO is showing the best performance on the basis laod balance factor by using the equation 7 .

\section{Conclusion}

In this paper, the Gray Wolf Optimizer based algorithm is implemented for the VANETs to make the optimized number of cluster. The results are illustrated in the graph with the comparative analysis of CLPSO and MOPSO. The outcomes show ICGWO is providing the optimized solution for the VANETs. This optimization lead us to reduce the resource requirements for the network. The number of hops required to deliver the packets will be reduced. Consequently, it will reduce the packet delays. All the factors together provide us the less routing cost. As, this algorithm is extracted from the social nature of gray wolves, so they have more aptitude to explore the search space to find the optimized target. The results are then compared with the popular algorithms (CLPSO and MOPSO). In future enhancement can occur in the algorithm to change the required objectives as per user demand. Other meta-heuristics i.e. Moth Flame Optimizer, Dragon Fly Optimizer, etc. can be implemented for the same problem.

\section{References}

[1] M. Fathian, G. R. Shiran, and A. R. Jafarian-Moghaddam, "Two new clustering algorithms for vehicular ad-hoc network based on ant colony system," Wireless Personal Communications, vol. 83, pp. 473-491, 2015. Article (CrossRef Link)

[2] K. Jothi and A. E. Jeyakumar, "Optimization and quality-of-service protocols in VANETs: a review," Artificial intelligence and evolutionary algorithms in engineering systems, ed: Springer, pp. 275-284, 2015. Article (CrossRef Link)

[3] A. Daeinabi, A. G. P. Rahbar, and A. Khademzadeh, "VWCA: An efficient clustering algorithm in vehicular ad hoc networks," Journal of Network and Computer Applications, vol. 34, pp. 207-222, 2011. Article (CrossRef Link)

[4] N. Kumar, N. Chilamkurti, and J. H. Park, "ALCA: agent learning-based clustering algorithm in vehicular ad hoc networks," Personal and ubiquitous computing, vol. 17, pp. 1683-1692, 2013. Article (CrossRef Link)

[5] P. Basu, N. Khan, and T. D. Little, "A mobility based metric for clustering in mobile ad hoc networks," in Proc. of Distributed computing systems workshop, 2001 international conference on, pp. 413-418, 2001. Article (CrossRef Link)

[6] Z. Y. Rawashdeh and S. M. Mahmud, "A novel algorithm to form stable clusters in vehicular ad hoc networks on highways," EURASIP Journal on Wireless Communications and Networking, vol. 2012, p. 15, 2012. Article (CrossRef Link)

[7] M. Gerla and J. T.-C. Tsai, "Multicluster, mobile, multimedia radio network," Wireless networks, vol. 1, pp. 255-265, 1995. Article (CrossRef Link)

[8] D. Turgut, S. K. Das, R. Elmasri, and B. Turgut, "Optimizing clustering algorithm in mobile ad hoc networks using genetic algorithmic approach," in Proc. of Global Telecommunications Conference, 2002. GLOBECOM'02. IEEE, pp. 62-66, 2002. Article (CrossRef Link) 
[9] I. I. Er and W. K. G. Seah, "Mobility-based d-hop clustering algorithm for mobile ad hoc networks," in Proc. of Wireless Communications and Networking Conference, 2004. WCNC. 2004 IEEE, pp. 2359-2364, 2004. Article (CrossRef Link)

[10] D. Aloise, A. Deshpande, P. Hansen, and P. Popat, "NP-hardness of Euclidean sum-of-squares clustering," Machine learning, vol. 75, pp. 245-248, 2009. Article (CrossRef Link)

[11] H. Ali, W. Shahzad, and F. A. Khan, "Energy-efficient clustering in mobile ad-hoc networks using multi-objective particle swarm optimization," Applied Soft Computing, vol. 12, pp. 1913-1928, 2012. Article (CrossRef Link)

[12] A. A. Abbasi and M. Younis, "A survey on clustering algorithms for wireless sensor networks," Computer communications, vol. 30, pp. 2826-2841, 2007. Article (CrossRef Link)

[13] M. Fahad, F. Aadil, S. Khan, P. A. Shah, K. Muhammad, J. Lloret, et al., "Grey wolf optimization based clustering algorithm for vehicular ad-hoc networks," Computers \& Electrical Engineering, 2018. Article (CrossRef Link)

[14] Y. Wang and F. Li, "Vehicular ad hoc networks," Guide to wireless ad hoc networks, ed: Springer, pp. 503-525, 2009. Article (CrossRef Link)

[15] U. Hernandez-Jayo, A. S. K. Mammu, and I. De-la-Iglesia, "Reliable Communication in Cooperative Ad hoc Networks," Contemporary Issues in Wireless Communications, ed: InTech, 2014. Article (CrossRef Link)

[16] P.-R. Sheu and C.-W. Wang, "A stable clustering algorithm based on battery power for mobile ad hoc networks," 淡江理工學刊, vol. 9, pp. 233-242, 2006. Article (CrossRef Link)

[17] S. Momeni and M. Fathy, "Clustering In VANETs," Intelligence for Nonlinear Dynamics and Synchronisation, ed: Springer, pp. 271-301, 2010. Article (CrossRef Link)

[18] W. Chen and S. Cai, "Ad hoc peer-to-peer network architecture for vehicle safety communications," IEEE Communications magazine, vol. 43, pp. 100-107, 2005. Article (CrossRef Link)

[19] Y.-T. Chang, J.-W. Ding, C.-H. Ke, and I.-Y. Chen, "A survey of handoff schemes for vehicular ad-hoc networks," in Proc. of Proceedings of the 6th international wireless communications and mobile computing conference, pp. 1228-1231, 2010. Article (CrossRef Link)

[20] M. Chatterjee, S. K. Das, and D. Turgut, "WCA: A weighted clustering algorithm for mobile ad hoc networks," Cluster computing, vol. 5, pp. 193-204, 2002. Article (CrossRef Link)

[21] W. Shahzad, F. A. Khan, and A. B. Siddiqui, "Clustering in mobile ad hoc networks using comprehensive learning particle swarm optimization (CLPSO)," Communication and Networking, ed: Springer, pp. 342-349, 2009. Article (CrossRef Link)

[22] L. Zhou, D. Wu, Z. Dong, and X. Li, "When Collaboration Hugs Intelligence: Content Delivery over Ultra-Dense Networks," IEEE Communications Magazine, vol. 55, pp. 91-95, 2017. Article (CrossRef Link)

[23] Z. Liang, D. Wu, J. Chen, and Z. Dong, "Greening the Smart Cities: Energy-Efficient Massive Content Delivery via D2D Communications," IEEE Transactions on Industrial Informatics, 2017. Article (CrossRef Link)

[24] H. Hu, Y. Wen, T.-S. Chua, J. Huang, W. Zhu, and X. Li, "Joint content replication and request routing for social video distribution over cloud CDN: A community clustering method," IEEE transactions on circuits and systems for video technology, vol. 26, pp. 1320-1333, 2016. Article (CrossRef Link)

[25] H. Hu, Y. Wen, T.-S. Chua, Z. Wang, J. Huang, W. Zhu, et al., "Community based effective social video contents placement in cloud centric CDN network," in Proc. of Multimedia and Expo (ICME), 2014 IEEE International Conference on, pp. 1-6, 2014. Article (CrossRef Link)

[26] F. Aadil, K. B. Bajwa, S. Khan, N. M. Chaudary, and A. Akram, "CACONET: Ant Colony Optimization (ACO) Based Clustering Algorithm for VANET," PloS one, vol. 11, p. e0154080, 2016. Article (CrossRef Link)

[27] F. Aadil, S. Khan, K. B. Bajwa, M. F. Khan, and A. Ali, "Intelligent Clustering in Vehicular ad hoc Networks," KSII Transactions on Internet \& Information Systems, vol. 10, 2016. Article (CrossRef Link) 
[28] D. Baker and A. Ephremides, "The architectural organization of a mobile radio network via a distributed algorithm," IEEE Transactions on communications, vol. 29, pp. 1694-1701, 1981. Article (CrossRef Link)

[29] R. Dewri, N. Poolsappasit, I. Ray, and D. Whitley, "Optimal security hardening using multi-objective optimization on attack tree models of networks," in Proc. of Proceedings of the 14th ACM conference on Computer and communications security, pp. 204-213, 2007. Article (CrossRef Link)

[30] M. Hadded, R. Zagrouba, A. Laouiti, P. Muhlethaler, and L. A. Saidane, "A multi-objective genetic algorithm-based adaptive weighted clustering protocol in vanet," in Proc. of Evolutionary Computation (CEC), 2015 IEEE Congress on, pp. 994-1002, 2015. Article (CrossRef Link)

[31] J. Kennedy, "Particle swarm optimization," Encyclopedia of machine learning, ed: Springer, pp. 760-766, 2011. Article (CrossRef Link)

[32] E. Emary, H. M. Zawbaa, and C. Grosan, "Experienced gray wolf optimization through reinforcement learning and neural networks," IEEE transactions on neural networks and learning systems, 2017. Article (CrossRef Link)

[33] L. D. Mech, "Alpha status, dominance, and division of labor in wolf packs," Canadian Journal of Zoology, vol. 77, pp. 1196-1203, 1999. Article (CrossRef Link) 


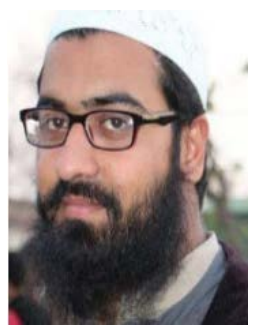

Muhammad Fahad Khan received his MS degree in Computer Science the Networks from COMSATS Institute of Information Technology, Attock, Pakistan, in 2018. He also did the BS in telecommunication and networking from the same institute. Currently he is working as Lecturer in the COMSATS Institute of Information Technology, Attock, Pakistan in the computer science department and pursing his $\mathrm{PhD}$. His current research interests include data mining, bio-informatics, and bio-inspired algorithms.

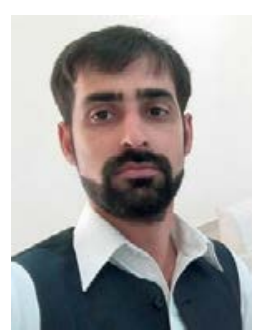

Farhan Aadil received his B.S. degree in Computer Science from Allama Iqbal Open University, Pakistan in 2005. He pursued a career in the computer science for 4 years (2005 to 2009). He received his M.S. degree in Software Engineering and $\mathrm{PhD}$ degree in Computer Engineering from University of Engineering and Technology, Taxila, Pakistan in 2011 and 2016 respectively. He is currently working as Assistant Professor in COMSATS Institute of Information Technology, Attock, Pakistan. His research interests include Vehicular ad hoc Networks, Intelligent Transportation Systems, and bio-inspired algorithms.

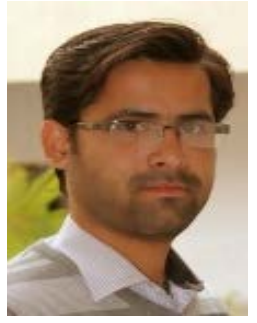

Muazzam Maqsood has done his Ph.D. in Software Engineering from University of Engineering and Technology, Taxila. He has completed his MS degree in 2013 from University of Engineering and Technology, Taxila. Currently, he is serving as a Assistant Professor in COMSATS Institute of Information Technology, Attock, Pakistan. His research interests include Machine Learning, Speech Processing, Recommender System and Image Processing.

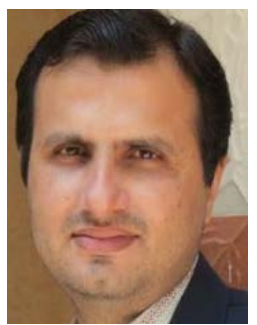

Salabat Khan received the B.S. degree in Computer Science from Virtual University, Lahore, Pakistan, in 2007, and the M.S. and PhD degree in computer science from the National University of Computer and Emerging Sciences, Islamabad, Pakistan, in 2009 and 2015 respectively. He is currently working as assistant professor in COMSATS Institute of Information Technology, Attock, Pakistan. His current research interests include data mining, pattern analysis, medical image processing, bio-informatics, and bio-inspired algorithms.

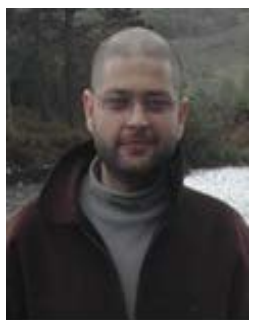

Bilal Haider is serving as Assistant Professor since 2013 in COMSATS Institute of Information Technology, Attock, Pakistan. He is a Ph.D. candidate in Computer Science at Bahria University Islamabad Campus. Bilal Haider received his degree of Masters of Science in Computer Science from Griffth College Dublin. He also served as Manager Information System in CIIT Attock Campus till 2017. His research interests include Vehicular ad hoc Networks, Intelligent Transportation Systems, Software Defined Networks. 